\title{
Systematical calculation of $\alpha$ decay half-lives with a generalized liquid drop model
}

\author{
Xiaojun Bao ${ }^{1}$, Hongfei Zhang ${ }^{1 *}$ Haifei Zhang ${ }^{1}$, G. Royer ${ }^{2}$, Junqing Li ${ }^{113}$
}

October 28, 2013

1. School of Nuclear Science and Technology, Lanzhou University, Lanzhou 730000, Peoples Republic of China

2. Laboratoire Subatech, UMR: IN2P3/CNRS-Université-Ecole des Mines, 44 Nantes, France

3. Institute of Modern Physics, Chinese Academy of Science, Lanzhou 730000, Peoples Republic of China

\begin{abstract}
A systematic calculation of $\alpha$ decay half-lives is presented for even-even nuclei between Te and $\mathrm{Z}=118$ isotopes. The potential energy governing $\alpha$ decay has been determined within a liquid drop model including proximity effects between the $\alpha$ particle and the daughter nucleus and adjusted to reproduce the experimental $\mathrm{Q}$ value. The $\alpha$ decay half-lives have been deduced from the WKB barrier penetration probability. The $\alpha$ decay half-lives obtained agree reasonably well with the experimental data.
\end{abstract}

Keywords: Preformation factor; $\alpha$ decay; Half-lives; Shell effects

*E-mail: zhanghongfei@lzu.edu.cn 


\section{Introduction}

The $\alpha$ decay is one of the most important decay channels of the heavy and superheavy nuclei. Measurements on the $\alpha$ decay can provide reliable information on the nuclear structure such as the ground state energy, the ground state half-life, the nuclear spin and parity, the nuclear deformation, the nuclear clustering, the shell effects, and the nuclear interaction [1-4]. Experimentally $\alpha$ decay of nuclei is used to identify the new nuclides and new elements through $\alpha$ decay chain from unknown parent nucleus to a known nuclide [5]. Recently, the interest in the $\alpha$ decay has been renewed because of the development of radioactive beams and new detector technology under low temperature. Some newly synthesized superheavy elements have recently been identified using $\alpha$ decay [6-9].

The process of $\alpha$ decay is fundamentally a quantum tunneling effect, which was first explained by Gamow and by Condon and Guerney in the 1920s [10,11]. Later on, theoretical calculations were performed to predict the absolute $\alpha$ decay width, to extract nuclear structure information, and to pursue a microscopic understanding of the $\alpha$ decay phenomenon. These studies are based on various theoretical models such as the shell-model, fission-like model, and cluster-model [12-27]. The simple empirical relations between $\alpha$ decay half-lives and decay energies are also discussed [28-31]. Generally tunneling penetration is used to describe the $\alpha$ decay, in which the penetration probability was calculated using WKB approximation assuming $\alpha$ particle tunneling through the potential barrier between $\alpha$ cluster and the daughter nucleus in the parent nucleus. In the unified fission approach $[32,33]$ the decay constant $\lambda$ is simply the product of the barrier penetrability $P$ and of a constant assault frequency $\nu_{0}$. Then, the height, position and width of the potential barriers are the main ingredients determining the half-lives. In the cluster-model $[12,34]$, the cluster is assumed to form before it penetrates the barrier and a preformation factor is included in the calculation. The decay constant $\lambda$ is defined as the product of the preformation factor, the assault frequency and the penetration probability. Usually, computing the $\alpha$ formation amplitude is a difficult task because the actual wave functions involved cannot be well defined. The $\alpha$ preformation factor is very important from the viewpoint of the nuclear structure. Numerous studies of the $\alpha$ decay have been concentrated on this problem $[35,36]$. In recent studies, the $\alpha$ preformation factor is extracted by dividing the experimental $\alpha$ decay width by the barrier penetration probability, which can easily be obtained from the WKB approximation $[25,37]$. 
The generalized liquid drop model (GLDM) was employed to calculate the nuclear potential. It has been successfully used to obtain the potential barriers for fusion reactions [38] and nuclear decays [39-41]. In our previous calculations [16], within a superasymmetric fission picture allows us to reproduce the experimental $\alpha$ decay half-lives when the experimental $\mathrm{Q}_{\alpha}$ values are used. The $\alpha$ decay half-lives have been calculated using the GLDM [16], in which the assault frequency $\nu_{0}$ is fixed as $1.0 \times 10^{20} \mathrm{~s}^{-1}$. In this work, the $\alpha$ decay halflives are calculated in the preformed cluster-model and the assault frequencies $\nu_{0}$ have been calculated using a classical method.

This paper is organized in the following way. In Section 2 we present the framework of the Generalized Liquid Drop Model. The numerical results are presented and discussed in Section 3. Finally, a brief summary of the present work is given in Section 4 .

\section{Theoretical framework}

The $\alpha$ decay constant is defined as,

$$
\lambda=P_{0} \nu_{0} P
$$

The assault frequency is calculated using the classical method,

$$
\nu_{0}=\frac{1}{2 R} \sqrt{\frac{2 E_{\alpha}}{M_{\alpha}}}
$$

where $R$ is the radius of the parent nucleus and $E_{\alpha}$ is the energy of the $\alpha$ particle, corrected for recoil; $M_{\alpha}$ being its mass.

The penetration probability $P$ is calculated within the WKB approximation. The GLDM energy of a deformed nucleus is defined as [38]:

$$
E=E_{V}+E_{S}+E_{C}+E_{\text {prox }}
$$

where the different terms are respectively the volume, surface, Coulomb, nuclear proximity energies.

For one-body shapes, the volume $E_{V}$, surface $E_{S}$ and Coulomb $E_{C}$ energies are given by

$$
\begin{gathered}
E_{V}=-15.494\left(1-1.8 I^{2}\right) A M e V, \\
E_{S}=17.9439\left(1-2.6 I^{2}\right) A^{2 / 3}\left(S / 4 \pi R_{0}^{2}\right) M e V,
\end{gathered}
$$




$$
E_{C}=0.6 e^{2}\left(Z^{2} / R_{0}\right) B_{C} M e V
$$

$B_{C}$ is the Coulomb shape dependent function, $S$ is the surface of the one body deformed nucleus and $I$ is the relative neutron excess.

$$
B_{C}=0.5 \int\left(V(\theta) / V_{0}\right)\left(R(\theta) / R_{0}\right)^{3} \sin \theta d \theta
$$

where $V(\theta)$ is the electrostatic potential at the surface and $V_{0}$ the surface potential of the sphere. The effective sharp radius $R_{0}$ has been chosen as

$$
R_{0}=1.28 A^{1 / 3}-0.76+0.8 A^{-1 / 3} \mathrm{fm} .
$$

This formula proposed in Ref. [42] is derived from the droplet model and the proximity energy and simulates rather a central radius for which $R_{0} / A^{1 / 3}$ increases slightly with the mass. It has been shown that this selected more elaborated expression can also be used to reproduce accurately the fusion, fission and cluster and alpha decay data. To ensure volume conservation, the radii $R_{1}$ and $R_{2}$ of the daughter and $\alpha$ nuclei are given by

$$
\begin{gathered}
R_{1}=R_{0}\left(1+\beta^{3}\right)^{-1 / 3}, \\
R_{2}=R_{0} \beta\left(1+\beta^{3}\right)^{-1 / 3},
\end{gathered}
$$

where,

$$
\beta=\frac{1.28 A_{1}^{1 / 3}-0.76+0.8 A_{1}^{-1 / 3}}{1.28 A_{2}^{1 / 3}-0.76+0.8 A_{2}^{-1 / 3}} .
$$

When the fragments are separated [43],

$$
\begin{gathered}
E_{V}=-15.494\left[\left(1-1.8 I_{1}^{2}\right) A_{1}+\left(1-1.8 I_{2}^{2}\right) A_{2}\right] \mathrm{MeV}, \\
E_{S}=17.9439\left[\left(1-2.6 I_{1}^{2}\right) A_{1}^{2 / 3}+\left(1-2.6 I_{2}^{2}\right) A_{2}^{2 / 3}\right] \mathrm{MeV}, \\
E_{C}=0.6 e^{2} Z_{1}^{2} / R_{1}+0.6 e^{2}\left(Z_{2}^{2} / R_{2}\right)+e^{2} Z_{1} Z_{2} / r \mathrm{MeV}
\end{gathered}
$$

The surface energy comes from the effects of the surface tension forces in a half space. When a neck or a gap appears between separated fragments an additional term called proximity energy must be added to take into account the effects of the nuclear forces between the close 
surface. It moves the barrier top to an external position and strongly decreases the pure Coulomb barrier:

$$
E_{\text {prox }}(r)=2 \gamma \int_{h_{\min }}^{h_{\max }} \Phi[D(r, h) / b] 2 \pi h d h
$$

where

$$
\gamma=0.9517 \sqrt{\left(1-2.6 I_{1}^{2}\right)\left(1-2.6 I_{2}^{2}\right)} \quad \mathrm{MeVfm}^{-2}
$$

$r$ is the distance between the mass centres, $h$ is the transverse distance varying from the neck radius or zero to the height of the neck border, $D$ is the distance between the opposite surfaces in consideration and $b$ is the surface width fixed at $0.99 \mathrm{fm}$. $\Phi$ is the proximity function. The surface parameter $\gamma$ is the geometric mean between the surface parameters of the two fragments.

The barrier penetrability $P$ is calculated within the action integral

$$
P=\exp \left[-\frac{2}{\hbar} \int_{R_{\text {in }}}^{R_{\text {out }}} \sqrt{2 B(r)(E(r)-E(\text { sphere }))} d r\right],
$$

with $E\left(R_{\text {in }}\right)=E\left(R_{\text {out }}\right)=Q_{\exp } . B(r)=\mu$, in which $\mu$ is the reduced mass.

To calculate the absolute $\alpha$ decay width, the $\alpha$ preformation factor $P_{\alpha}$ is indispensable based on the Gamow picture, which measures the probability that an $\alpha$ cluster is present in the decaying nucleus. Within a superasymmetric fission picture the preformation factor $P_{0}$ has been taken as 1 in previous studies and that allows us to reproduce the experimental $\alpha$ decay half-lives when the experimental $\mathrm{Q}_{\alpha}$ values are used. However, there are still small differences between the calculated and experimental values and these discrepancies may be used to determine the $\alpha$ preformation probability. The microscopic calculation gives a value of 0.3 for the $\alpha$ cluster preformation factor of even-even nucleus ${ }^{212} \mathrm{Po}$ [12]. However, development on the microscopical description of the $\alpha$ cluster preformation factor is still slow due to the complexity of the nuclear many-body problem. Experiments have shown that the preformation factor varies smoothly in the open shell region and has a value smaller than 1.0 [1]. As a result, it is reasonable and appropriate to take the preformation factor as a constant for all even-even nuclei. This means both the medium mass $\alpha$ emitters and heavy ones can be well described in a consistent way by the GLDM. We fix the value of the preformation factors $P_{\alpha}=0.38$ for even-even nuclei, which is consistent with both the microscopic calculations and the experimental data of open shell nuclei $[1,12]$. Finally, the partial half-life is related to the decay constant $\lambda$ by $T_{1 / 2}=\ln 2 / \lambda$. 


\section{Results and discussions}
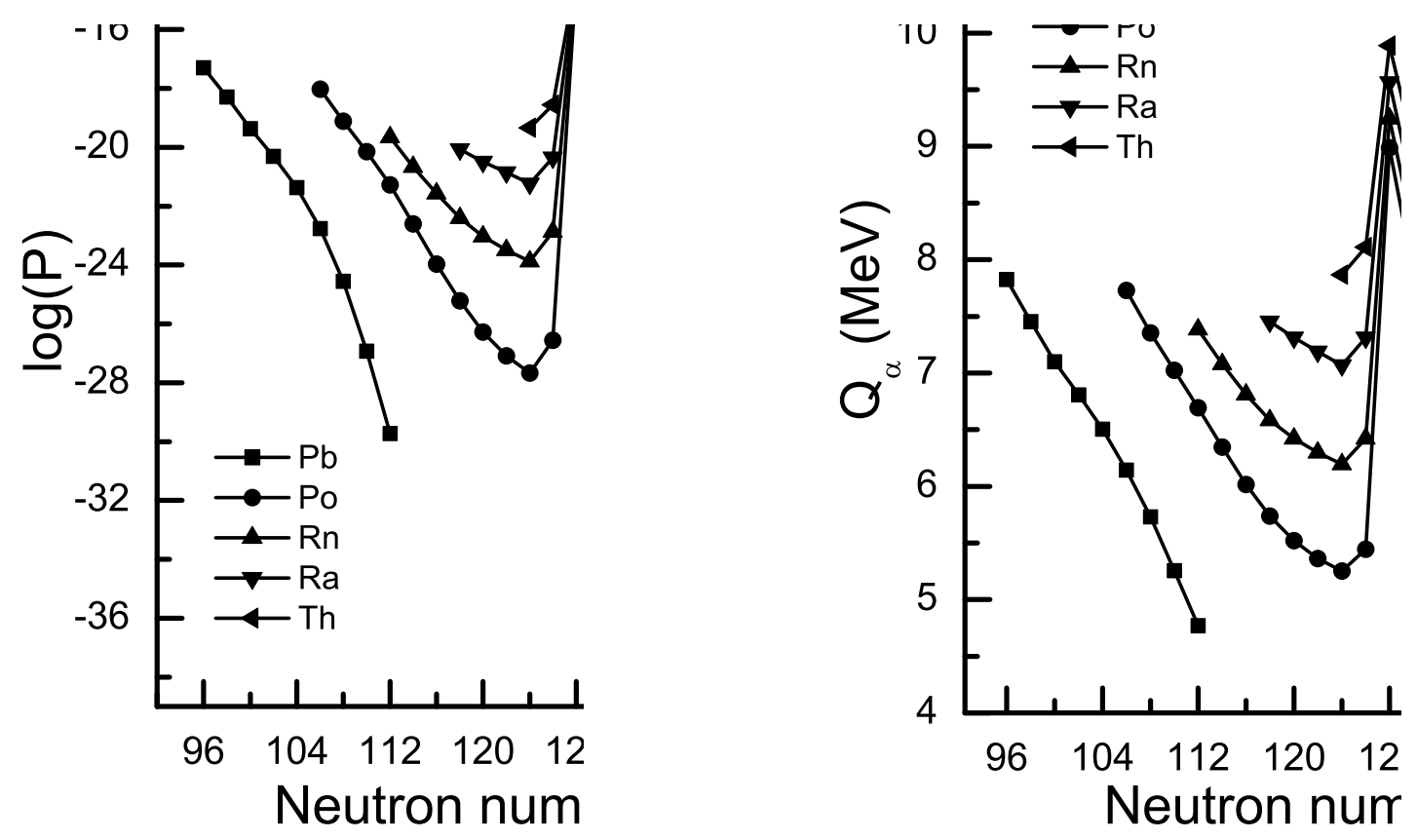

Figure 1: The penetration probabilities and experimental decay energies $\mathrm{Q}_{\alpha}$ of $\alpha$ decay even-even $\mathrm{Pb}, \mathrm{Po}, \mathrm{Rn}, \mathrm{Ra}$ and Th isotopes.

The half-lives of $\alpha$ decay from the ground state to ground state for even-even nuclei with proton number $\mathrm{Z}=52-118$ have been calculated within the GLDM. The ground state spin and parity of all even-even nuclei is $0^{+}$. This means that the $\alpha$ decay of even-even nuclei mainly proceeds to the ground state of the daughter nucleus. Actually, the parent nucleus can also decay to the excited states of the daughter nucleus, this probability is very small in normal cases, and it can be neglected for a systematic calculation of half-lives. The numerical results for the even-even nuclei from Te to 118 are listed in Table 1 . The first and second columns denote the parent nucleus and neutron number $\mathrm{N}$, respectively. The third and forth columns are respectively the experimental decay energies and partial half-lives of $\alpha$ decay. The calculated half-lives are listed in the last column. The meanings of columns 6-10 are similar to those of columns 1-5. It is known experimentally that the magnitude of $\alpha$ decay half-lives of the even-even nuclei varies in a very wide range from $10^{-7}$ to $10^{22} \mathrm{~s}$. Although the amplitude of the variation of half-lives is as high as $10^{29}$ times, we can see from Table 1 that the experimental $\alpha$ decay half-lives of many even-even nuclei are 
reproduced within a factor of 3 by GLDM. But for the four regions the vicinity of $\mathrm{N}=50$, $\mathrm{N}=126, \mathrm{~N}=152$ and superheavy region the deviations between experimental and calculated values are relatively large. The big deviation occurring for Te isotope chains are mainly due to the shell effect ${ }^{100} \mathrm{Sn}$ nucleus. In the superheavy nuclei region, one can see from the Table 1 that the calculated values deviate obviously from the experimental ones. This may be understood as a consequence of the following two sides. On the theoretical side, the superheavy nuclei $\alpha$ preformation factor is smaller than the one of medium and heavy nuclei [25] in our previous study. Theoretical calculation should give better agreement with the experimental data if the preformation factor is considered as a variable with different parent nuclei. On the experimental side, it would be interesting to improve the precision of experimental $\alpha$ decay half-lives.

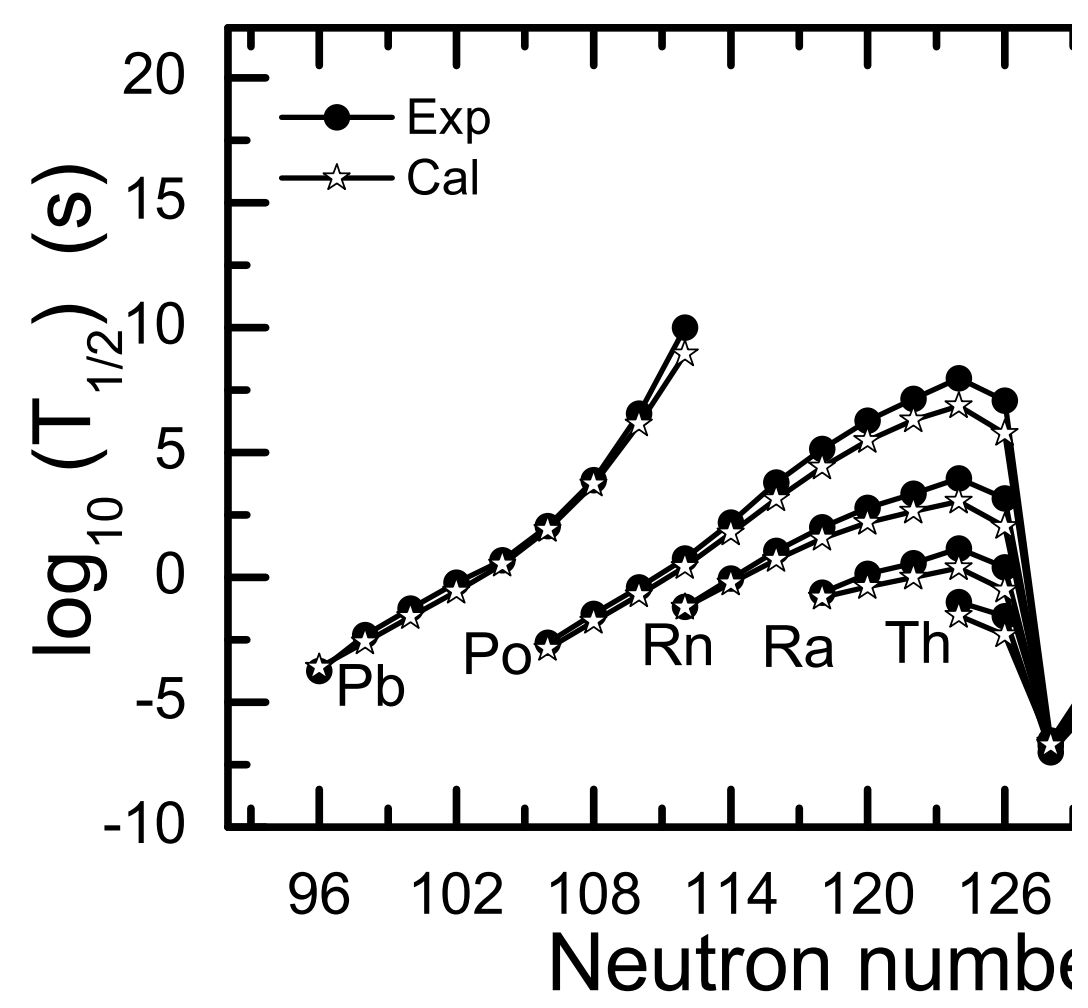

Figure 2: Comparison of the calculated $\alpha$ decay half-lives with the experimental data for even-even nuclei ranging from $Z=82$ to $Z=90$, showing the shell effect at the neutron magic numbers $\mathrm{N}=126$.

Fig.1 shows the penetration probability in the left hand side as a function of neutron number N. The penetration probabilities decrease with increasing neutron number up to the spherical shell closure $\mathrm{N}=126$, and then increase rapidly with the neutron number. The maximum probability appears at $\mathrm{N}=128$ for all the four selected isotopes. For the nuclei 
Table 1: Comparison between experimental and theoretical $\alpha$ decay half-lives and of the even-even nuclei with proton number $\mathrm{Z}=52-118$. The units of the $\alpha$ decay energies and half-lives are $\mathrm{MeV}$ and second respectively, and the experimental results are taken from the recently data $[44,45]$.

\begin{tabular}{|c|c|c|c|c|c|c|c|c|c|}
\hline Nucleus & $\mathrm{N}$ & Q & $T_{1 / 2}(\exp )$. & $T_{1 / 2}$ (cal.) & Nucleus & $\mathrm{N}$ & Q & $T_{1 / 2}(\exp )$. & $T_{1 / 2}$ (cal.) \\
\hline${ }_{52}^{106} \mathrm{Te}$ & 54 & 4.290 & $8.0 \times 10^{-5}$ & $65.0 \times 10^{-5}$ & ${ }_{76}^{162} \mathrm{Os}$ & 86 & 6.767 & $2.1 \times 10^{-3}$ & $3.6 \times 10^{-3}$ \\
\hline${ }_{52}^{108} \mathrm{Te}$ & 56 & 3.420 & $4.3 \times 10^{0}$ & $28.5 \times 10^{0}$ & ${ }_{76}^{164} \mathrm{Os}$ & 88 & 6.479 & $4.1 \times 10^{-3}$ & $31.4 \times 10^{-3}$ \\
\hline${ }_{52}^{110} \mathrm{Te}$ & 58 & 2.699 & $6.2 \times 10^{5}$ & $99.0 \times 10^{5}$ & ${ }_{76}^{166} \mathrm{Os}$ & 90 & 6.139 & $3.0 \times 10^{-1}$ & $4.9 \times 10^{-1}$ \\
\hline${ }_{54}^{110} \mathrm{Xe}$ & 56 & 3.875 & $3.9 \times 10^{0}$ & $0.87 \times 10^{0}$ & ${ }_{76}^{168} \mathrm{Os}$ & 92 & 5.816 & $4.9 \times 10^{0}$ & $9.1 \times 10^{0}$ \\
\hline${ }_{54}^{112} \mathrm{Xe}$ & 58 & 3.330 & $3.0 \times 10^{2}$ & $20.3 \times 10^{2}$ & ${ }_{76}^{170} \mathrm{Os}$ & 94 & 5.537 & $7.8 \times 10^{1}$ & $13.9 \times 10^{1}$ \\
\hline${ }_{62}^{146} \mathrm{Sm}$ & 84 & 2.528 & $2.2 \times 10^{15}$ & $12.7 \times 10^{15}$ & ${ }_{76}^{172} \mathrm{Os}$ & 96 & 5.224 & $1.7 \times 10^{3}$ & $3.9 \times 10^{3}$ \\
\hline${ }_{64}^{148} \mathrm{Gd}$ & 84 & 3.271 & $2.2 \times 10^{9}$ & $6.4 \times 10^{9}$ & ${ }_{76}^{174} \mathrm{Os}$ & 98 & 4.870 & $1.8 \times 10^{5}$ & $2.8 \times 10^{5}$ \\
\hline${ }_{64}^{150} \mathrm{Gd}$ & 86 & 2.808 & $5.6 \times 10^{13}$ & $22.0 \times 10^{13}$ & ${ }_{76}^{186} \mathrm{Os}$ & 110 & 2.820 & $6.3 \times 10^{22}$ & $10.4 \times 10^{22}$ \\
\hline${ }_{66}^{150} \mathrm{Dy}$ & 84 & 4.351 & $1.2 \times 10^{3}$ & $2.4 \times 10^{3}$ & ${ }_{78}^{166} \mathrm{Pt}$ & 88 & 7.286 & $3.0 \times 10^{-4}$ & $4.5 \times 10^{-4}$ \\
\hline${ }_{66}^{152} \mathrm{Dy}$ & 86 & 3.726 & $8.6 \times 10^{6}$ & $25.3 \times 10^{6}$ & ${ }_{78}^{168} \mathrm{Pt}$ & 90 & 6.990 & $2.0 \times 10^{-3}$ & $3.3 \times 10^{-3}$ \\
\hline${ }_{66}^{154} \mathrm{Dy}$ & 88 & 2.945 & $9.5 \times 10^{13}$ & $19.6 \times 10^{13}$ & ${ }_{78}^{170} \mathrm{Pt}$ & 92 & 6.707 & $6.0 \times 10^{-3}$ & $26.4 \times 10^{-3}$ \\
\hline${ }_{68}^{152} \mathrm{Er}$ & 84 & 4.934 & $1.1 \times 10^{1}$ & $2.1 \times 10^{1}$ & ${ }_{78}^{172} \mathrm{Pt}$ & 94 & 6.464 & $1.0 \times 10^{-1}$ & $1.6 \times 10^{-1}$ \\
\hline${ }_{68}^{154} \mathrm{Er}$ & 86 & 4.279 & $4.8 \times 10^{4}$ & $7.4 \times 10^{4}$ & ${ }_{78}^{174} \mathrm{Pt}$ & 96 & 6.183 & $1.2 \times 10^{0}$ & $1.7 \times 10^{0}$ \\
\hline${ }_{68}^{156} \mathrm{Er}$ & 88 & 3.483 & $6.7 \times 10^{9}$ & $38.5 \times 10^{9}$ & ${ }_{78}^{176} \mathrm{Pt}$ & 98 & 5.885 & $1.6 \times 10^{1}$ & $2.6 \times 10^{1}$ \\
\hline${ }_{70}^{154} \mathrm{Yb}$ & 84 & 5.474 & $4.4 \times 10^{-1}$ & $6.9 \times 10^{-1}$ & ${ }_{78}^{178} \mathrm{Pt}$ & 100 & 5.573 & $2.7 \times 10^{2}$ & $5.8 \times 10^{2}$ \\
\hline${ }_{70}^{156} \mathrm{Yb}$ & 86 & 4.811 & $2.6 \times 10^{2}$ & $8.7 \times 10^{2}$ & ${ }_{78}^{180} \mathrm{Pt}$ & 102 & 5.240 & $1.9 \times 10^{4}$ & $2.3 \times 10^{4}$ \\
\hline${ }_{70}^{158} \mathrm{Yb}$ & 88 & 4.170 & $4.3 \times 10^{6}$ & $4.5 \times 10^{6}$ & ${ }_{78}^{182} \mathrm{Pt}$ & 104 & 4.951 & $4.2 \times 10^{5}$ & $7.9 \times 10^{5}$ \\
\hline${ }_{72}^{156} \mathrm{Hf}$ & 84 & 6.028 & $2.4 \times 10^{-2}$ & $3.5 \times 10^{-2}$ & ${ }_{78}^{184} \mathrm{Pt}$ & 106 & 4.629 & $5.9 \times 10^{7}$ & $10.0 \times 10^{7}$ \\
\hline${ }_{72}^{158} \mathrm{Hf}$ & 86 & 5.404 & $6.4 \times 10^{0}$ & $10.9 \times 10^{0}$ & ${ }_{78}^{186} \mathrm{Pt}$ & 108 & 4.598 & $5.3 \times 10^{9}$ & $7.1 \times 10^{9}$ \\
\hline${ }_{72}^{160} \mathrm{Hf}$ & 88 & 4.902 & $1.9 \times 10^{3}$ & $2.7 \times 10^{3}$ & ${ }_{78}^{188} \mathrm{Pt}$ & 110 & 4.320 & $3.3 \times 10^{12}$ & $1.6 \times 10^{12}$ \\
\hline${ }_{72}^{162} \mathrm{Hf}$ & 90 & 4.416 & $4.9 \times 10^{5}$ & $14.8 \times 10^{5}$ & ${ }_{78}^{190} \mathrm{Pt}$ & 112 & 3.252 & $2.0 \times 10^{19}$ & $1.8 \times 10^{19}$ \\
\hline${ }_{74}^{158} \mathrm{~W}$ & 84 & 6.613 & $1.3 \times 10^{-3}$ & $2.0 \times 10^{-3}$ & ${ }_{80}^{172} \mathrm{Hg}$ & 92 & 7.524 & $2.3 \times 10^{-4}$ & $3.9 \times 10^{-4}$ \\
\hline${ }_{74}^{160} \mathrm{~W}$ & 86 & 6.065 & $1.1 \times 10^{-1}$ & $1.6 \times 10^{-1}$ & ${ }_{80}^{174} \mathrm{Hg}$ & 94 & 7.233 & $2.0 \times 10^{-3}$ & $2.6 \times 10^{-3}$ \\
\hline${ }_{74}^{162} \mathrm{~W}$ & 88 & 5.677 & $3.0 \times 10^{0}$ & $5.9 \times 10^{0}$ & ${ }_{80}^{176} \mathrm{Hg}$ & 96 & 6.899 & $2.3 \times 10^{-2}$ & $2.9 \times 10^{-2}$ \\
\hline${ }_{74}^{164} \mathrm{~W}$ & 90 & 5.279 & $1.7 \times 10^{2}$ & $3.1 \times 10^{2}$ & ${ }_{80}^{178} \mathrm{Hg}$ & 98 & 6.577 & $5.0 \times 10^{-1}$ & $3.5 \times 10^{-1}$ \\
\hline${ }_{74}^{166} \mathrm{~W}$ & 92 & 4.856 & $5.5 \times 10^{4}$ & $4.1 \times 10^{4}$ & ${ }_{80}^{180} \mathrm{Hg}$ & 100 & 6.258 & $5.4 \times 10^{0}$ & $5.2 \times 10^{0}$ \\
\hline${ }_{74}^{168} \mathrm{~W}$ & 94 & 4.500 & $1.6 \times 10^{6}$ & $4.6 \times 10^{6}$ & ${ }_{80}^{182} \mathrm{Hg}$ & 102 & 5.996 & $7.8 \times 10^{1}$ & $5.6 \times 10^{1}$ \\
\hline
\end{tabular}




\begin{tabular}{|c|c|c|c|c|c|c|c|c|c|}
\hline Nucleus & $\mathrm{N}$ & Q & $T_{1 / 2}(\exp )$. & $T_{1 / 2}$ (cal.) & Nucleus & $\mathrm{N}$ & Q & $T_{1 / 2}$ (exp.) & $T_{1 / 2}($ cal. $)$ \\
\hline${ }_{80}^{184} \mathrm{Hg}$ & 104 & 5.662 & $2.8 \times 10^{3}$ & $1.6 \times 10^{3}$ & ${ }_{86}^{200} \mathrm{Rn}$ & 114 & 7.044 & $1.2 \times 10^{0}$ & $0.83 \times 10^{0}$ \\
\hline${ }_{80}^{186} \mathrm{Hg}$ & 106 & 5.204 & $5.0 \times 10^{5}$ & $3.0 \times 10^{5}$ & ${ }_{86}^{202} \mathrm{Rn}$ & 116 & 6.773 & $1.2 \times 10^{1}$ & $0.71 \times 10^{1}$ \\
\hline${ }_{80}^{188} \mathrm{Hg}$ & 108 & 4.703 & $5.2 \times 10^{8}$ & $2.4 \times 10^{8}$ & ${ }_{86}^{204} \mathrm{Rn}$ & 118 & 6.546 & $1.0 \times 10^{2}$ & $0.49 \times 10^{2}$ \\
\hline${ }_{82}^{178} \mathrm{~Pb}$ & 96 & 7.790 & $2.3 \times 10^{-4}$ & $3.0 \times 10^{-4}$ & ${ }_{86}^{206} \mathrm{Rn}$ & 120 & 6.384 & $5.5 \times 10^{2}$ & $2.1 \times 10^{2}$ \\
\hline${ }_{82}^{180} \mathrm{~Pb}$ & 98 & 7.419 & $4.2 \times 10^{-3}$ & $3.2 \times 10^{-3}$ & ${ }_{86}^{208} \mathrm{Rn}$ & 122 & 6.261 & $2.4 \times 10^{3}$ & $0.65 \times 10^{3}$ \\
\hline${ }_{82}^{182} \mathrm{~Pb}$ & 100 & 7.066 & $5.5 \times 10^{-2}$ & $3.9 \times 10^{-2}$ & ${ }_{86}^{210} \mathrm{Rn}$ & 124 & 6.159 & $9.0 \times 10^{3}$ & $1.6 \times 10^{3}$ \\
\hline${ }_{82}^{184} \mathrm{~Pb}$ & 102 & 6.774 & $6.1 \times 10^{-1}$ & $3.6 \times 10^{-1}$ & ${ }_{86}^{212} \mathrm{Rn}$ & 126 & 6.385 & $1.4 \times 10^{3}$ & $0.15 \times 10^{3}$ \\
\hline${ }_{82}^{186} \mathrm{~Pb}$ & 104 & 6.470 & $1.2 \times 10^{1}$ & $0.44 \times 10^{1}$ & ${ }_{86}^{214} \mathrm{Rn}$ & 128 & 9.208 & $2.7 \times 10^{-7}$ & $2.9 \times 10^{-7}$ \\
\hline${ }_{82}^{188} \mathrm{~Pb}$ & 106 & 6.109 & $2.8 \times 10^{2}$ & $1.1 \times 10^{2}$ & ${ }_{86}^{216} \mathrm{Rn}$ & 130 & 8.197 & $4.5 \times 10^{-5}$ & $8.2 \times 10^{-5}$ \\
\hline${ }_{82}^{190} \mathrm{~Pb}$ & 108 & 5.697 & $1.8 \times 10^{4}$ & $0.76 \times 10^{4}$ & ${ }_{86}^{218} \mathrm{Rn}$ & 132 & 7.263 & $3.5 \times 10^{-2}$ & $5.3 \times 10^{-2}$ \\
\hline${ }_{82}^{192} \mathrm{~Pb}$ & 110 & 5.221 & $3.5 \times 10^{6}$ & $2.0 \times 10^{6}$ & ${ }_{86}^{220} \mathrm{Rn}$ & 134 & 6.405 & $5.6 \times 10^{1}$ & $8.8 \times 10^{1}$ \\
\hline${ }_{82}^{194} \mathrm{~Pb}$ & 112 & 4.738 & $8.8 \times 10^{9}$ & $1.4 \times 10^{9}$ & ${ }_{86}^{222} \mathrm{Rn}$ & 136 & 5.590 & $3.3 \times 10^{5}$ & $6.3 \times 10^{5}$ \\
\hline${ }_{82}^{210} \mathrm{~Pb}$ & 128 & 3.792 & $3.7 \times 10^{16}$ & $2.0 \times 10^{16}$ & ${ }_{88}^{206} \mathrm{Ra}$ & 118 & 7.415 & $2.4 \times 10^{-1}$ & $2.1 \times 10^{-1}$ \\
\hline${ }_{84}^{190} \mathrm{Po}$ & 106 & 7.693 & $2.5 \times 10^{-3}$ & $1.7 \times 10^{-3}$ & ${ }_{88}^{208} \mathrm{Ra}$ & 120 & 7.273 & $1.3 \times 10^{0}$ & $0.57 \times 10^{0}$ \\
\hline${ }_{84}^{192} \mathrm{Po}$ & 108 & 7.320 & $3.4 \times 10^{-2}$ & $2.2 \times 10^{-2}$ & ${ }_{88}^{210} \mathrm{Ra}$ & 122 & 7.152 & $3.8 \times 10^{0}$ & $1.3 \times 10^{0}$ \\
\hline${ }_{84}^{194} \mathrm{Po}$ & 110 & 6.987 & $3.9 \times 10^{-1}$ & $2.5 \times 10^{-1}$ & ${ }_{88}^{212} \mathrm{Ra}$ & 124 & 7.032 & $1.4 \times 10^{1}$ & $0.33 \times 10^{1}$ \\
\hline${ }_{84}^{196} \mathrm{Po}$ & 112 & 6.658 & $5.7 \times 10^{0}$ & $3.6 \times 10^{0}$ & ${ }_{88}^{214} \mathrm{Ra}$ & 126 & 7.273 & $2.5 \times 10^{0}$ & $0.41 \times 10^{0}$ \\
\hline${ }_{84}^{198} \mathrm{Po}$ & 114 & 6.309 & $1.9 \times 10^{2}$ & $0.81 \times 10^{2}$ & ${ }_{88}^{216} \mathrm{Ra}$ & 128 & 9.526 & $1.8 \times 10^{-7}$ & $2.5 \times 10^{-7}$ \\
\hline${ }_{84}^{200} \mathrm{Po}$ & 116 & 5.981 & $6.2 \times 10^{3}$ & $2.0 \times 10^{3}$ & ${ }_{88}^{218} \mathrm{Ra}$ & 130 & 8.546 & $2.5 \times 10^{-5}$ & $4.7 \times 10^{-5}$ \\
\hline${ }_{84}^{202} \mathrm{Po}$ & 118 & 5.701 & $1.4 \times 10^{5}$ & $0.38 \times 10^{5}$ & ${ }_{88}^{220} \mathrm{Ra}$ & 132 & 7.592 & $1.8 \times 10^{-2}$ & $2.5 \times 10^{-2}$ \\
\hline${ }_{84}^{204} \mathrm{Po}$ & 120 & 5.485 & $1.9 \times 10^{6}$ & $0.45 \times 10^{6}$ & ${ }_{88}^{222} \mathrm{Ra}$ & 134 & 6.679 & $3.4 \times 10^{1}$ & $4.5 \times 10^{1}$ \\
\hline${ }_{84}^{206} \mathrm{Po}$ & 122 & 5.327 & $1.4 \times 10^{7}$ & $0.30 \times 10^{7}$ & ${ }_{88}^{224} \mathrm{Ra}$ & 136 & 5.789 & $3.2 \times 10^{5}$ & $5.1 \times 10^{5}$ \\
\hline${ }_{84}^{208} \mathrm{Po}$ & 124 & 5.215 & $9.1 \times 10^{7}$ & $0.12 \times 10^{7}$ & ${ }_{88}^{226} \mathrm{Ra}$ & 138 & 4.871 & $5.0 \times 10^{10}$ & $14.6 \times 10^{10}$ \\
\hline${ }_{84}^{210} \mathrm{Po}$ & 126 & 5.407 & $1.2 \times 10^{7}$ & $0.092 \times 10^{7}$ & ${ }_{90}^{214} \mathrm{Th}$ & 124 & 7.827 & $8.7 \times 10^{-2}$ & $0.04 \times 10^{-2}$ \\
\hline${ }_{84}^{212} \mathrm{Po}$ & 128 & 8.954 & $3.0 \times 10^{-7}$ & $2.5 \times 10^{-7}$ & ${ }_{90}^{216} \mathrm{Th}$ & 126 & 8.072 & $2.6 \times 10^{-2}$ & $0.62 \times 10^{-2}$ \\
\hline${ }_{84}^{214} \mathrm{Po}$ & 130 & 7.833 & $1.6 \times 10^{-4}$ & $1.6 \times 10^{-4}$ & ${ }_{90}^{218} \mathrm{Th}$ & 128 & 9.849 & $1.2 \times 10^{-7}$ & $2.2 \times 10^{-7}$ \\
\hline${ }_{84}^{216} \mathrm{Po}$ & 132 & 6.906 & $1.5 \times 10^{-1}$ & $1.5 \times 10^{-1}$ & ${ }_{90}^{220} \mathrm{Th}$ & 130 & 8.953 & $9.7 \times 10^{-6}$ & $20.0 \times 10^{-6}$ \\
\hline${ }_{84}^{218} \mathrm{Po}$ & 134 & 6.115 & $1.9 \times 10^{2}$ & $2.1 \times 10^{2}$ & ${ }_{90}^{222} \mathrm{Th}$ & 132 & 8.127 & $2.1 \times 10^{-3}$ & $3.0 \times 10^{-3}$ \\
\hline${ }_{86}^{198} \mathrm{Rn}$ & 112 & 7.349 & $6.6 \times 10^{-2}$ & $8.1 \times 10^{-2}$ & ${ }_{90}^{224} \mathrm{Th}$ & 134 & 7.298 & $1.1 \times 10^{0}$ & $1.3 \times 10^{0}$ \\
\hline
\end{tabular}




\begin{tabular}{|c|c|c|c|c|c|c|c|c|c|}
\hline Nucleus & $\mathrm{N}$ & Q & $T_{1 / 2}$ (exp.) & $T_{1 / 2}$ (cal.) & Nucleus & $\mathrm{N}$ & Q & $T_{1 / 2}$ (exp.) & $T_{1 / 2}$ (cal.) \\
\hline${ }_{90}^{226} \mathrm{Th}$ & 136 & 6.451 & $1.2 \times 10^{3}$ & $2.8 \times 10^{3}$ & ${ }_{98}^{242} \mathrm{Cf}$ & 144 & 7.517 & $2.6 \times 10^{2}$ & $1.7 \times 10^{2}$ \\
\hline${ }_{90}^{228} \mathrm{Th}$ & 138 & 5.520 & $6.0 \times 10^{7}$ & $12.9 \times 10^{7}$ & ${ }_{98}^{244} \mathrm{Cf}$ & 146 & 7.329 & $1.2 \times 10^{3}$ & $0.82 \times 10^{3}$ \\
\hline${ }_{90}^{230} \mathrm{Th}$ & 140 & 4.770 & $2.4 \times 10^{12}$ & $8.7 \times 10^{12}$ & ${ }_{98}^{246} \mathrm{Cf}$ & 148 & 6.862 & $1.3 \times 10^{5}$ & $0.64 \times 10^{5}$ \\
\hline${ }_{90}^{232} \mathrm{Th}$ & 142 & 4.082 & $4.4 \times 10^{17}$ & $33.1 \times 10^{17}$ & ${ }_{98}^{248} \mathrm{Cf}$ & 150 & 6.361 & $2.9 \times 10^{7}$ & $1.4 \times 10^{7}$ \\
\hline${ }_{92}^{222} \mathrm{U}$ & 130 & 9.430 & $1.5 \times 10^{-6}$ & $6.3 \times 10^{-6}$ & ${ }_{98}^{250} \mathrm{Cf}$ & 152 & 6.128 & $4.1 \times 10^{8}$ & $2.3 \times 10^{8}$ \\
\hline${ }_{92}^{224} \mathrm{U}$ & 132 & 8.620 & $9.4 \times 10^{-4}$ & $6.2 \times 10^{-4}$ & ${ }_{98}^{252} \mathrm{Cf}$ & 154 & 6.217 & $8.6 \times 10^{7}$ & $7.3 \times 10^{7}$ \\
\hline${ }_{92}^{226} \mathrm{U}$ & 134 & 7.701 & $2.7 \times 10^{-1}$ & $3.2 \times 10^{-1}$ & ${ }_{98}^{254} \mathrm{Cf}$ & 156 & 5.927 & $1.7 \times 10^{9}$ & $2.9 \times 10^{9}$ \\
\hline${ }_{92}^{228} \mathrm{U}$ & 136 & 6.803 & $5.7 \times 10^{2}$ & $6.1 \times 10^{2}$ & ${ }_{100}^{246} \mathrm{Fm}$ & 146 & 78 & $1.3 \times 10^{0}$ & $0.93 \times 10^{0}$ \\
\hline${ }_{92}^{230} \mathrm{U}$ & 138 & 5.993 & $1.7 \times 10^{6}$ & $3.1 \times 10^{6}$ & ${ }_{100}^{248} \mathrm{Fm}$ & 148 & 8.002 & $3.8 \times 10^{1}$ & $1.5 \times 10^{1}$ \\
\hline${ }_{92}^{232} \mathrm{U}$ & 140 & 5.414 & $2.2 \times 10^{9}$ & $5.1 \times 10^{9}$ & ${ }_{100}^{250} \mathrm{Fm}$ & 150 & 7.557 & $1.8 \times 10^{3}$ & $0.54 \times 10^{3}$ \\
\hline${ }_{92}^{234} \mathrm{U}$ & 142 & 4.858 & $7.7 \times 10^{13}$ & $2.4 \times 10^{13}$ & ${ }_{100}^{252} \mathrm{Fm}$ & 152 & 7.153 & $9.1 \times 10^{4}$ & $2.1 \times 10^{4}$ \\
\hline${ }_{92}^{236} \mathrm{U}$ & 144 & 4.573 & $7.4 \times 10^{14}$ & $32.0 \times 10^{14}$ & ${ }_{100}^{254} \mathrm{Fm}$ & 154 & 7.308 & $1.2 \times 10^{4}$ & $0.42 \times 10^{4}$ \\
\hline${ }_{92}^{238} \mathrm{U}$ & 146 & 4.270 & $1.4 \times 10^{17}$ & $10.5 \times 10^{17}$ & ${ }_{100}^{256} \mathrm{Fm}$ & 156 & 7.027 & $1.2 \times 10^{5}$ & $0.60 \times 10^{5}$ \\
\hline${ }_{94}^{228} \mathrm{Pu}$ & 134 & 7.940 & $2.1 \times 10^{0}$ & $0.29 \times 10^{0}$ & ${ }_{102}^{252} \mathrm{No}$ & 150 & 8.550 & $4.1 \times 10^{0}$ & $1.1 \times 10^{0}$ \\
\hline${ }_{94}^{232} \mathrm{Pu}$ & 138 & 6.716 & $1.8 \times 10^{4}$ & $0.91 \times 10^{4}$ & ${ }_{102}^{254} \mathrm{No}$ & 152 & & $5.7 \times 10^{1}$ & $1.1 \times 10^{1}$ \\
\hline${ }_{94}^{234} \mathrm{Pu}$ & 140 & 6.310 & $5.3 \times 10^{5}$ & $6.1 \times 10^{5}$ & ${ }_{102}^{256} \mathrm{No}$ & 154 & 8.581 & $2.9 \times 10^{0}$ & $0.7 \times 10^{0}$ \\
\hline${ }_{94}^{236} \mathrm{Pu}$ & 142 & 5.867 & $9.0 \times 10^{7}$ & $11.2 \times 10^{7}$ & ${ }_{104}^{254} \mathrm{Rf}$ & 150 & 9.210 & $1.7 \times 10^{-1}$ & $0.60 \times 10^{-1}$ \\
\hline${ }_{94}^{238} \mathrm{Pu}$ & 144 & 5.593 & $2.8 \times 10^{9}$ & $3.7 \times 10^{9}$ & ${ }_{104}^{256} \mathrm{Rf}$ & 152 & 8.930 & $2.1 \times 10^{0}$ & $0.34 \times 10^{0}$ \\
\hline${ }_{94}^{240} \mathrm{Pu}$ & 146 & 5.256 & $2.1 \times 10^{11}$ & $4.7 \times 10^{11}$ & ${ }_{104}^{258} \mathrm{Rf}$ & 154 & 9.190 & $1.1 \times 10^{-1}$ & $0.55 \times 10^{-1}$ \\
\hline${ }_{94}^{242} \mathrm{Pu}$ & 148 & 4.985 & $1.2 \times 10^{13}$ & $3.2 \times 10^{13}$ & ${ }_{106}^{260} \mathrm{Sg}$ & 154 & 9.920 & $1.2 \times 10^{-2}$ & $0.29 \times 10^{-2}$ \\
\hline${ }_{94}^{244} \mathrm{Pu}$ & 150 & 4.666 & $2.5 \times 10^{15}$ & $7.5 \times 10^{15}$ & ${ }_{106}^{266} \mathrm{Sg}$ & 160 & 880 & $3.3 \times 10^{1}$ & $0.15 \times 10^{1}$ \\
\hline${ }_{96}^{238} \mathrm{Cm}$ & 142 & 6.670 & $7.9 \times 10^{4}$ & $8.5 \times 10^{4}$ & ${ }_{108}^{264} \mathrm{Hs}$ & 56 & 91 & $1.1 \times 10^{-3}$ & $0.25 \times 10^{-3}$ \\
\hline${ }_{96}^{240} \mathrm{Cm}$ & 144 & 6.398 & $2.3 \times 10^{6}$ & $1.5 \times 10^{6}$ & ${ }_{108}^{266} \mathrm{Hs}$ & 158 & 10.346 & $3.1 \times 10^{-3}$ & $0.86 \times 10^{-3}$ \\
\hline${ }_{96}^{242} \mathrm{Cm}$ & 146 & 6.216 & $1.4 \times 10^{7}$ & $1.1 \times 10^{7}$ & ${ }_{110}^{270} \mathrm{Ds}$ & 160 & 11.117 & $2.1 \times 10^{-4}$ & $0.57 \times 10^{-4}$ \\
\hline${ }_{96}^{244} \mathrm{Cm}$ & 148 & 5.902 & $5.7 \times 10^{8}$ & $5.2 \times 10^{8}$ & & 172 & 10.370 & $1.4 \times 10^{-1}$ & $0.24 \times 10^{-1}$ \\
\hline${ }_{96}^{246} \mathrm{Cm}$ & 150 & 5.475 & $1.5 \times 10^{11}$ & $1.8 \times 10^{11}$ & $\begin{array}{l}288 \\
114\end{array}$ & 174 & 10.072 & $7.5 \times 10^{-1}$ & $1.2 \times 10^{-1}$ \\
\hline${ }_{96}^{248} \mathrm{Cm}$ & 152 & 5.162 & $1.2 \times 10^{13}$ & $2.0 \times 10^{13}$ & ${ }_{116}^{290} \mathrm{Lv}$ & 174 & 10.990 & $8.0 \times 10^{-3}$ & $2.7 \times 10^{-3}$ \\
\hline${ }_{96}^{250} \mathrm{Cm}$ & 154 & 5.169 & $1.5 \times 10^{12}$ & $17.6 \times 10^{12}$ & ${ }_{116}^{292} \mathrm{Lv}$ & 176 & 10.774 & $2.4 \times 10^{-2}$ & $0.79 \times 10^{-2}$ \\
\hline${ }_{98}^{240} \mathrm{Cf}$ & 142 & 7.719 & $4.1 \times 10^{1}$ & $3.5 \times 10^{1}$ & $\begin{array}{l}294 \\
118 \\
\end{array}$ & 176 & 11.810 & $1.4 \times 10^{-3}$ & $0.14 \times 10^{-3}$ \\
\hline
\end{tabular}


with two neutrons outside the closed shell, the $\alpha$ particle emission is easier than that of the other nuclei of the same isotopes. The closed shell structures play also the key role for the penetrability mechanism. Moreover, the values of the maximum penetration probabilities are nearly identical at $\mathrm{N}=128$ for $\mathrm{Po}, \mathrm{Rn}, \mathrm{Ra}$, and $\mathrm{Th}$ isotopes, after which the penetration probability decreases again with increasing N. At the same neutron number the penetration probability increases with increasing proton number because proton number of Po isotopes are closer to a proton magic number $\mathrm{Z}=82$ than those of $\mathrm{Rn}, \mathrm{Ra}$, and $\mathrm{Th}$. The closer the nucleon number is to a magic number, the more difficult the $\alpha$ particle penetrates from the parent nuclei. The experimental decay energy $Q_{\alpha}$ is shown in the right hand side also as a function of $\mathrm{N}$ in Fig.1. One can see from the figure that $\mathrm{Q}_{\alpha}$ decreases with the increasing neutron number $\mathrm{N}$ for the $\mathrm{Pb}, \mathrm{Po}, \mathrm{Rn}, \mathrm{Ra}$, and $\mathrm{Th}$ isotopes before the neutron number $\mathrm{N}=126$. The $\mathrm{Q}_{\alpha}$ values increase sharply after $\mathrm{N}=126$ and reach the maximum at $\mathrm{N}=128$, then they decrease again quickly for $\mathrm{Po}, \mathrm{Rn}, \mathrm{Ra}$ and Th isotopes. The changes in $\mathrm{Q}_{\alpha}$ and penetration probability $\mathrm{P}$ are similar.

In order to show the systematic behavior of the agreement between model and data clearly, we also plot the comparison of experimental half-lives and theoretical ones in Fig.2. The theoretical points (stars) almost coincide with the experimental ones (black circles). First, Po, Rn, Ra and Th isotope chains half-lives are show in Fig.2 as a function of neutron number N. On the one hand, the half-lives increase with increasing neutron number up to the spherical shell closure $\mathrm{N}=126$, and then decrease rapidly with the neutron number. The minimum half-lives appears at $\mathrm{N}=128$ for all the four selected isotopes, indicating $\alpha$ emission is much easier for the nuclei with two neutrons outside the shell closure. Moreover, the values of the minimum half-lives are nearly identical at $\mathrm{N}=128$, after which the half-lives increase again with increasing N. On the other hand, in Fig.2 there is a clear decrease in the half-life before the neutron number $\mathrm{N}=128$, shown in the Po, Rn, Ra and Th isotopes. This is attributed to the strong $\mathrm{N}=126$ shell effect: the main effect of the $\mathrm{N}=126$ shell is included in the decay $\mathrm{Q}$ value, which is closely related to the nuclear structure, and the remaining effect is largely absorbed into the $\alpha$ preformation factor. Because the constant preformation factor cannot completely describe the detailed features of nuclear structure, the strong shell effects are clearly shown from the increased deviations in the neighborhood of $N=126$. At the same neutron number, half-lives decrease with increasing proton number because proton number of $\mathrm{Po}$ isotopes are closer to a proton magic number $\mathrm{Z}=82$ than those of $\mathrm{Rn}$, $\mathrm{Ra}$ 
and Th. Both $\mathrm{N}=126$ and $\mathrm{Z}=82$ are well known as magic numbers. The closer the nucleon number is to shell closure, the more difficultly the $\alpha$ cluster forms in the parent nuclei. The dramatic change of the half-lives around the magic number indicates that the shell effects play an important role on $\alpha$ formation mechanism in the parent nuclei. There is no doubt that the theoretical calculation should give better agreement with the experimental data if the preformation factor is considered as a variable with different parent nuclei instead of a constant, especially for the closed shell region nuclei.
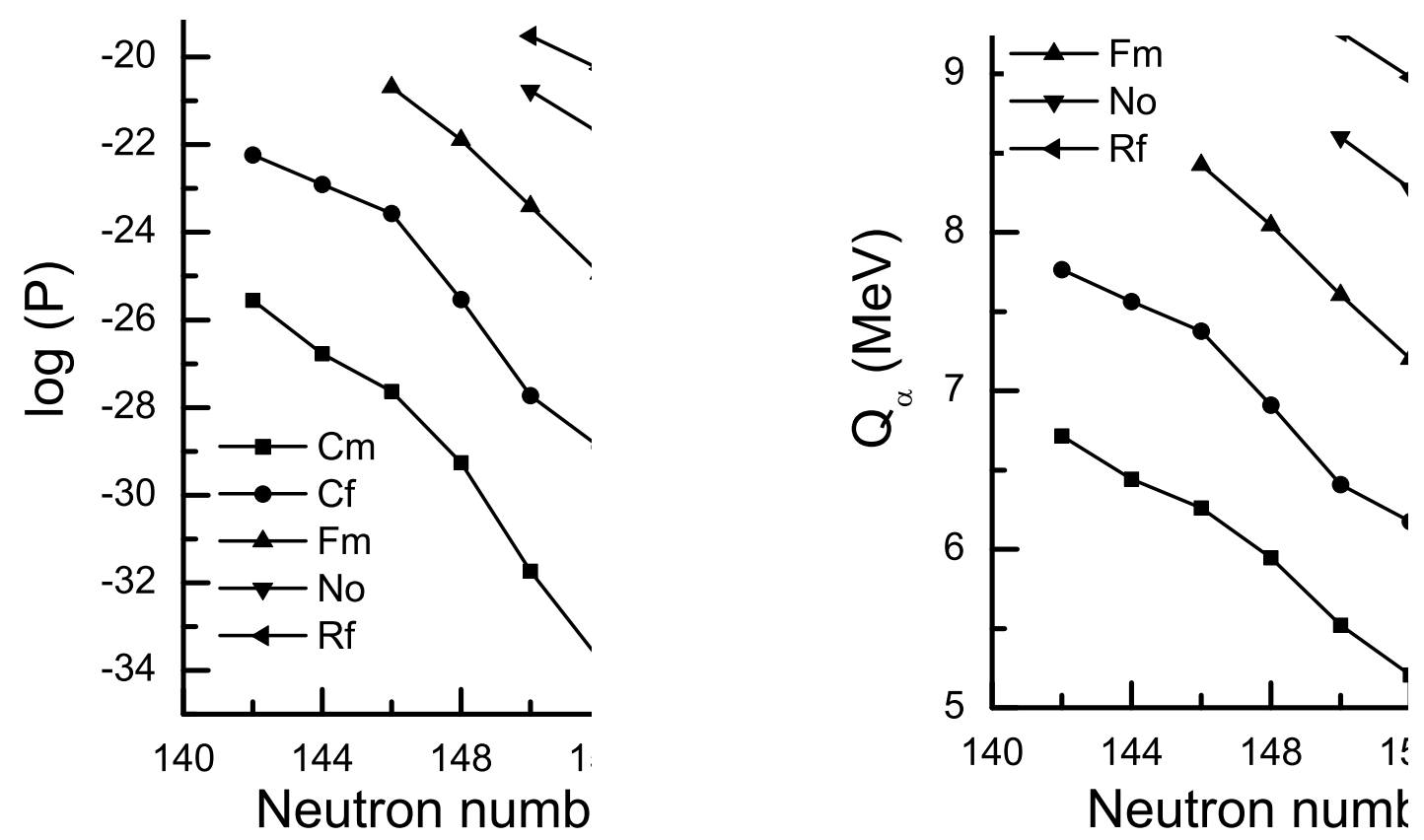

Figure 3: The penetration probabilities and experimental released energies $\mathrm{Q}_{\alpha}$ of $\alpha$ decay even-even Cm, Cf, Fm, No and Rf isotopes.

The penetration probabilities and the $\mathrm{Q}_{\alpha}$ values for $\mathrm{Cm}, \mathrm{Cf}, \mathrm{Fm}$, No and Rf isotopes are also shown in Fig.3. We can see from this figure that the change in $\mathrm{Q}_{\alpha}$ is similar to that in penetration probability. They decrease with increasing neutron number up to $\mathrm{N}=152$, then increase with $\mathrm{N}$ up to $\mathrm{N}=154$. After that, they decrease again. It is well known that the shell effects for $\alpha$ radioactivity are related to the $\mathrm{Q}_{\alpha}$ value, which is maximum when the daughter nuclei has a magic number of neutrons and protons. The changes in $\mathrm{Q}_{\alpha}$ and $\mathrm{P}$ are similar. The lower is $\mathrm{Q}_{\alpha}$ the more difficult it will be for an $\alpha$ cluster to penetrate the potential barrier in the parent nucleus in accordance with quantum tunneling penetration theory. 
The calculated half-lives are compared with the experimental ones for isotopes of Th, U, $\mathrm{Pu}, \mathrm{Cm}, \mathrm{Cf}, \mathrm{Fm}, \mathrm{No}$ and Rf in Fig.4. We can see from this figure that the experimental data are well reproduced by GLDM in these isotopes considered here. The theoretical points (stars) almost coincide with the experimental ones (black circles). Fig.4 shows that the $\alpha$ decay half-lives decrease as the value of $\mathrm{Z}$ increases, which reflects the stability gained by these nuclei when two protons are removed. The half-lives do not increase monotonously with $\mathrm{N}$ and a maximum exists at $\mathrm{N}=152$ due to the shell effects. We may conclude that $\mathrm{N}=152$ is a deformed magic number. Around the shell $\mathrm{N}=152$, the variation of half-lives is approximately 10 times for Cm, Cf, Fm and No isotopic chains. For instance, the $\alpha$ decay half-life of ${ }^{250} \mathrm{Cf}(\mathrm{N}=152)$ is only ten times larger than that of ${ }^{252} \mathrm{Cf}(\mathrm{N}=154)$. This demonstrates that the influence of the deformed shell on half-lives is less than that of the spherical one.

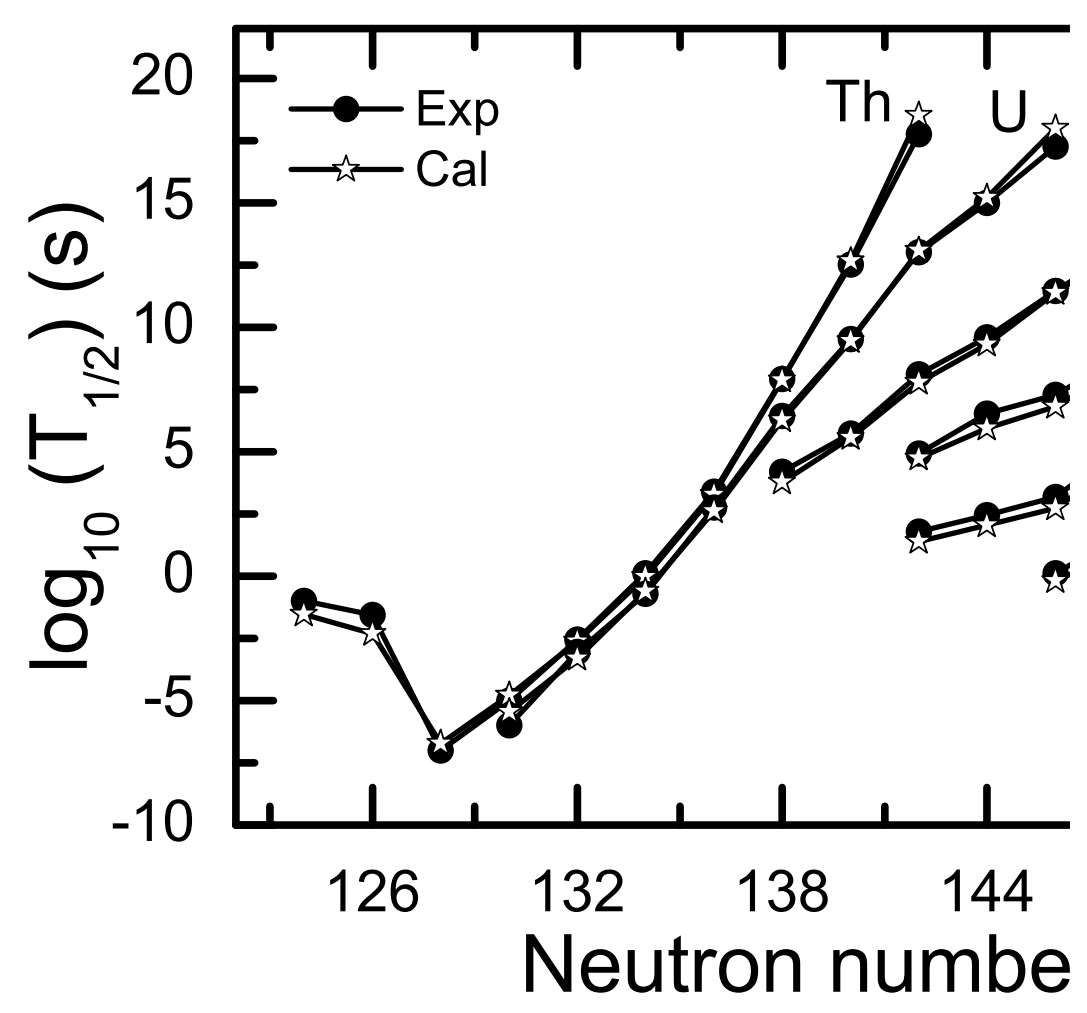

Figure 4: Comparison of the calculated $\alpha$ decay half-lives with the experimental data for even-even nuclei ranging from $\mathrm{Z}=90$ to $\mathrm{Z}=104$, showing the shell effect at the neutron magic number $\mathrm{N}=152$. 


\section{Summary}

A global calculation of the $\alpha$ decay half-lives are presented for the even-even nuclei from $\mathrm{Z}=52$ to $\mathrm{Z}=118$, in which the penetration probability was calculated using WKB approximation assuming $\alpha$ particle tunneling through the potential barrier between $\alpha$ cluster and the daughter nucleus in the parent nucleus. The barriers are constructed with the GLDM and the decay energies used in our calculations are extracted from experimental data. The assault frequency $\nu_{0}$ has been calculated using a classical method. The value of the preformation factors $\mathrm{P}_{\alpha}$ has been fixed to 0.38 for even-even nuclei. Finally, the decay constant is the product of the preformation, the penetration, and the assault frequency. The obtained decay half-lives agree reasonably well with the available experimental data. The change in $\alpha$ decay half-lives with neutron number shows that shell effects play an important role in the behavior of $\alpha$ decay half-lives around magic numbers. It is worth noting that the aim of this work is not only to reproduce the experimental data, but also to extend our understanding of $\alpha$ decay half-lives around shell closures.

\section{Acknowledgements}

The work is supported by the Natural Science Foundation of China (Grants 11175074, 11105035 and 10975064), the Fundamental Research Funds for the Central Universities (grants lzujbky-2012-5), by the CAS Knowledge Innovation Project NO.KJCX-SYW-N02.

\section{References}

[1] P.E. Hodgson and E. Batak, Phys. Rep. 374 (2003) 1.

[2] R. Lovas, R. Liotta, A. Insolia, K. Varga, and D. Delion, Phys. Rep. 294 (1998) 265.

[3] W. Seif, Phys. Rev. C 74 (2006) 034302.

[4] D. Delion, S. Peltonen, and J. Suhonen, Phys. Rev. C 73 (2006) 014315.

[5] S. Hofmann and G. Münzenberg, Rev. Mod. Phys. 72 (2000) 733.

[6] K. Gregorich, et al., Phys. Rev. C 74 (2006) 044611. 
[7] J. Dvorak, et al., Phys. Rev. Lett 97 (2006) 242501.

[8] Y.T. Oganessian et al., Phys. Rev. C 69 (2004) 054607;

Y.T. Oganessian, et al., Phys. Rev. C 74 (2006) 044602;

Y.T. Oganessian, et al., Phys. Rev. C 76 (2007) 011601;

Y.T. Oganessian, et al., Phys. Rev. C 87 (2013) 034605.

[9] P. Sahu, et al., Phys. Rev. C 72 (2005) 034604.

[10] G.Z. Gamov, Phys. 51 (1928) 204.

[11] R.W. Gurney and E. U. Condon, Nature 122 (1928) 439.

[12] K. Varga, R. Lovas, and R. Liotta, Phys. Rev. Lett 99 (1992) 37.

[13] B. A. Brown, Phys. Rev. C 46 (1992) 811.

[14] B. Buck, et al., At. Data and Nucl. Data Tables 54 (1993) 53.

[15] B. Buck, A. Merchant, and S. Perez, Phys. Rev. C 51 (1995) 559.

[16] G. Royer, J. Phys.G: Nucl. Part. Phys. 26 (2000) 1149.

[17] G. Royer and R. Gherghescu, Nucl. Phys. A 699 (2002) 479 .

[18] S. Duarte, et al., At. Data and Nucl. Data Tables 80 (2002) 235.

[19] P. Mohr, Phy. Rev. C 73 (2006) 031301.

[20] P. R. Chowdhury, C. Samanta, and D. Basu, Phy. Rev. C 73 (2006) 014612.

[21] C. Xu and Z. Ren, Phy. Rev. C 74 (2006) 014304.

[22] D.N. Poenaru, R.A. Gherghescu, and W. Greiner, Phy. Rev. C 85 (2012) 034615; D.N. Poenaru, R.A. Gherghescu, and W. Greiner, Phys. Rev. Lett. 107 (2011) 062503.

[23] C. Qi, F. Xu, R. J. Liotta, and R. Wyss, Phys. Rev. Lett 103 (2009) 072501; C. Qi, A.N. Andreyev, M. Huyse, R.J. Liotta, P. Van Duppen, and R.A. Wyss, Phys. Rev. C 81 (2010) 064319.

[24] G. Royer and H.F. Zhang, Phys. Rev. C 77 (2008) 037602.

[25] H.F. Zhang and G. Royer, Phys. Rev. C 77 (2008) 054318. 
[26] D. Ni and Z. Ren, Phys. Rev. C 80 (2009) 051303.

[27] H.F. Zhang, Y. Gao, N. Wang, J.Q. Li, E.G. Zhao, and G. Royer, Phys. Rev. C 85 (2012) 014325..

[28] D. Ni, Z. Ren, T. Dong, and C. Xu, Phys. Rev. C 78 (2008) 044310.

[29] D. Delion, Phys. Rev. C 80 (2009) 024310.

[30] V.Y. Denisov and A. Khudenko, Phys. Rev. C 79 (2009) 054614.

[31] D. Poenaru, R. Gherghescu, and W. Greiner, J. Phys. G: Nucl. Part. Phys. 39 (2012) 015105; D.N. Poenaru, R.A. Gherghescu, and W. Greiner, Phy. Rev. C 83 (2011) 014601.

[32] D. N. Poenaru, W. Greiner, M. Ivascu, and A. Sandulescu, Phys. Rev. C 32 (1985) 2198.

[33] G. Royer, R. K. Gupta, and V. Y. Denisov, Nucl. Phys. A 632 (1998) 275.

[34] S. Malik and R. K. Gupta, Phys. Rev. C 39 (1989) 1992.

[35] Y. Gambhir, P. Ring, and P. Schuck, Phys. Rev. Lett. 51 (1983) 1235.

[36] G. Röpke, A. Schnell, P. Schuck, and P. Nozieres, Phys. Rev. Lett. 80 (1998) 3177.

[37] G. Zhang, X. Le, and H. Zhang, Phys. Rev. C 80 (2009) 064325.

[38] G. Royer and B. Remaud, Nucl. Phys. A 444 (1985) 477.

[39] X J Bao, H F Zhang, B S Hu, et al., J. Phys.G: Nucl. Part. Phys. 39 (2012) 095103.

[40] X J Bao, H F Zhang, G. Royer, et al., Nucl. Phys. A 906 (2013) 1-13.

[41] H F Zhang, Y J Wang, J M Dong, J Q Li, W Scheid, J. Phys. G: Nucl. Part. Phys. 37 (2010) 085107.

[42] J. Blocki, J. Randrup, W. Swiatecki, and C. Tsang, Annals of Physics 105 (1977) 427.

[43] H.F. Zhang, W. Zuo, J.Q. Li, and G. Royer, Phys. Rev. C 74 (2006) 017304.

[44] G. Audi, F.G. Kondev, M. Wang, B. Pfeiffer, X. Sun, J. Blachot and M. MacCormick, Chinese Physics C 36 (2012) 1157. 
[45] M. Wang, G. Audi, A.H. Wapstra, F.G. Kondev, M. MacCormick, X. Xu and B. Pfeiffer, Chinese Physics C 36 (2012) 1603. 